\title{
Author Correction: The genome sequence of allopolyploid Brassica juncea and analysis of differential homoeolog gene expression influencing selection
}

Jinghua Yang, Dongyuan Liu, Xiaowu Wang, Changmian Ji, Feng Cheng, Baoning Liu, Zhongyuan Hu, Sheng Chen, Deepak Pental, Youhui Ju, Pu Yao, Xuming Li, Kun Xie, Jianhui Zhang, Jianlin Wang, Fan Liu, Weiwei Ma, Jannat Shopan, Hongkun Zheng, Sally A Mackenzie and Mingfang Zhang

Correction to: Nature Genetics https://doi.org/10.1038/ng.3657, published online 5 September 2016.

Following publication of this article, the authors have corrected 426 chimeric scaffolds in this genome (total scaffold number 10,684). The genome assembly has now been improved as V1.5, and the updated genome assembly is available to be downloaded from http://brassicadb.org/brad/datasets/pub/Genomes/Brassica_juncea/V1.5/.

Published online: 24 September 2018

https://doi.org/10.1038/s41588-018-0227-4

\section{Author Correction: The rate of meiotic gene conversion varies by sex and age}

Bjarni V Halldorsson, Marteinn T Hardarson, Birte Kehr, Unnur Styrkarsdottir, Arnaldur Gylfason, Gudmar Thorleifsson, Florian Zink, Adalbjorg Jonasdottir, Aslaug Jonasdottir, Patrick Sulem, Gisli Masson, Unnur Thorsteinsdottir, Agnar Helgason, Augustine Kong, Daniel F Gudbjartsson and Kari Stefansson

Correction to: Nature Genetics https://doi.org/10.1038/ng.3669, published online 19 September 2016.

In the version of this article published, statements about the impact of insertions and deletions on gene conversions were incorrect. We reported a bias toward deletions, whereas in fact the bias was toward insertions. We are deeply indebted to Laurent Duret and Brice Letcher for noticing this mistake in our manuscript. The following statements are incorrect in the published manuscript.

Abstract:

Published text: "For indels, we demonstrate a $65.6 \%$ preference for the shorter allele."

Correct text: "For indels, we demonstrate a $65.6 \%$ preference for the longer allele."

Results:

Published text: "Our results showed a bias of $65.6 \%(95 \% \mathrm{CI}=53.3-76.6 \%, P=0.018$; Table 3$)$ toward the shorter allele for indels in allelic gene conversions."

Correct text: "Our results showed a bias of $65.6 \%(95 \% \mathrm{CI}=53.3-76.6 \%, P=0.018$; Table 3$)$ toward the longer allele for indels in allelic gene conversions."

Table 3:

Published text: "Deletion bias (\%)"

Correct text: "Insertion bias (\%)"

Supplementary information:

Published text: "We observe a bias towards the shorter alleles of 65.6\% (95\% CI 53.2-77.4, p: 0.018) in the set of gene converted indels (cf.Table 3).

Of the 61 gene converted indels, 40 were deletions from the reference genome, while 21 were insertions into the reference genome. Deletion bias was estimated as 65\% (95\% CI 50.0- 78.9) for indels that are deletions from the reference genome and 66.7\% (95\% CI 42.1-85.7), for indels that are insertions into the reference genome. No difference was found in deletion bias between those markers that are deletions vs. those that are insertions (p-value: 0.95 ).

Thirty of the 61 gene converted indels represented the deletion or insertion of one base-pair. No significant difference could be found in deletion bias by indel length."

Correct text: "We observe a bias towards the longer alleles of 65.6\% (95\% CI 53.2-77.4, p: 0.018) in the set of gene converted indels (cf.Table 3). Of the 61 gene converted indels, 40 were deletions from the reference genome, while 21 were insertions into the reference genome. Insertion bias was estimated as 65\% (95\% CI 50.0- 78.9) for indels that are deletions from the reference genome and 66.7\% (95\% CI 42.1-85.7), for indels that are insertions into the reference genome. No difference was found in insertion bias between those markers that are deletions vs. those that are insertions (p-value: 0.95 ).

Thirty of the 61 gene converted indels represented the deletion or insertion of one base-pair. No significant difference could be found in insertion bias by indel length."

Published online: 20 September 2018

https://doi.org/10.1038/s41588-018-0228-3 